\title{
Statistiken zum Mathematikstudium
}

\author{
Britta Berndtsen, Miriam Dieter, Günter Törner
}

Mittlerweile sind beinahe acht Jahre ins Land gezogen, seitdem wir uns zuletzt in den Mitteilungen der DMV ([7]) umfassend mit Anfänger-, Studierenden- und Absolventenzahlen in den mathematischen Studiengängen beschäftigt haben. Inzwischen wurde die Bachelor-MasterReform umgesetzt, wodurch sich die Studiengänge und ihre inhaltlichen Orientierungen wesentlich verändert haben.

Wir beginnen damit, dass wir über die Anfängerzahlen des Jahres 2014 berichten. Ältere Arbeiten kann man von der von den Autoren eingerichteten Internetseite herunterladen. Leser, die sich mit den interessanten Statistiken eigenständig beschäftigen wollen, verweisen wir auf die im Literaturverzeichnis zitierten Publikationen des Statistischen Bundesamtes. In der Rubrik Bildung und Kultur gibt es seit 2003 die Reihen Studierende an Hochschulen und Prüfungen an Hochschulen. Auch die Broschüren Hochschulen auf einen Blick $(2012,2013,2016)$ enthalten wichtige Informationen.

\section{Auswirkungen der Bologna-Reform}

\section{I.I Die unübersichtliche Vielfalt der Studiengänge}

Aufgrund der Bologna-Reform sind in fast allen Bundesländern die Diplomstudiengänge ausgelaufen. Von 67I Studiengängen an I54 Universitäten (staatlich, privat und kirchlich) werden nur noch 42 mit dem Abschluss Diplom angeboten ${ }^{2}$. In der Mathematik lassen lediglich die Universität Leipzig und die Technische Universität Bergakademie Freiberg im Einklang mit dem sächsischen Hochschulgesetz noch Diplomstudiengänge zu; in Leipzig kann ein Diplomgrad in Mathematik oder Wirtschaftsmathematik und in Freiberg ein solcher in der Angewandten Mathematik erworben werden. Das Auslaufen der Diplomstudiengänge ab 2013 hat deutlich gemacht, dass noch viele Langzeitstudierende unterwegs waren; insgesamt 740 Diplomprüfungen verzeichnet die Statistik von Destatis im Jahr 2014, ${ }^{3}$ immerhin noch rund ein Drittel der akademischen Abschlüsse im Studienbereich Mathematik mit zum Teil extrem langen Studienzeiten.

Bereits in unseren früheren Erhebungen hatten wir erkannt, dass wir die Zahlen für die Lehramtsstudiengänge strikt von den für uns interessanten Zahlen der anderen Studiengänge trennen sollten. Diese Trennung ist nicht zuletzt aufgrund der proklamierten Polyvalenz des Bachelor-Studiums schwieriger geworden. Die folgende Liste belegt, dass die Palette der Studiengänge nahezu unüberschaubar geworden sind. Das Statistische Bundesamt (Destatis) ([3], S. I I 2 f) differenziert die Studienabsolventen im Studienbereich Mathematik im Prüfungsjahr 2014 nach folgenden 46 (!) Abschlüssen:
- Universitärer Abschluss (ohne Lehramtsprüfungen)

Diplom $(U)$

Diplom I (U-GH)

- Promotionen

Promotion (Abschlussprüfung vorausgesetzt)

Strukturiertes Promotionsstudium

Promotion nach FH-Abschluss bzw. wiss. Kurzstudium

- Bachelor-/Master-Abschlüsse für das Lehramt an Grund- und Hauptschulen/Primarstufe

- LA Bachelor Grundschulen

LA Bachelor Hauptschulen

LA Grundstufe/Primarstufen

LA Grund- und Hauptschulen

LA Grundschulen

LA Master Grundschulen

LA Master Grundstufe/Primarstufe

LA Master Grund- und Hauptschulen

- Bachelor-/Master-Abschlüsse für das Lehramt Sekundarstufe I/ Grundschule, Primarstufe (stufenübergreifend)

- LA Bachelor Sekundarstufe I/Primarstufe

LA Bachelor Grundschulen/Sekundarstufe I

LA Master Sekundarstufe I/Primarstufe

LA Master Grundschulen/Sekundarstufe I

- Bachelor-/Master-Abschlüsse für das Lehramt an Realschulen/Sekundarstufe I

- LA Realschulen

LA Haupt- und Realschulen/ Unterstufe und Mittelstufe Gymnasien

LA Bachelor Realschulen

LA Bachelor Haupt- und Realschulen/Unterstufe und Mittelstufe Gymnasien

LA Mittelstufe/Sekundarstufe I

LA Master Realschulen

- LA Master Haupt- und Realschulen/Unterstufe und Mittelstufe Gymnasien

LA Master Mittelstufe/Sekundarstufe I

- Bachelor-/Master-Abschlüsse für das Lehramt in der Sekundarstufe II/I (stufenübergreifend)

LA Sekundarstufe II/Sekundarstufe I

- Lehramts-, Bachelor- und Masterprüfungen an Gymnasien/

Sekundarstufe II, allgemeinbildende Schulen

LA Gymnasien

LA Bachelor Gymnasien

LA Master Oberstufe/Sekundarstufe II, allgemeinbildende Schulen

LA Master Gymnasien

- Bachelor-/Master-Abschlüsse für das Lehramt an Sonder- und Förderschulen

- LA Sonderschulen/Förderschulen

LA Bachelor Sonderschulen/Förderschulen

LA Master Sonderschulen/Förderschulen

- BSc-/MSc-Abschlüsse für das Lehramt an Beruflichen Schulen

LA Berufliche Schulen

LA Bachelor Berufliche Schulen

LA Master Berufliche Schulen

- LA Bachelor

LA Bachelor (soweit keine Differenzierung möglich)

- LA Master

LA Master (soweit keine Differenzierung möglich)

- Fachhochschulabschluss Diplom (FH)

- Bachelorabschluss

Mehr-Fächer-Bachelor mit Lehramtsoption

Mehr-Fächer-Bachelor ohne Lehramtsoption

Bachelor an Universitäten

Bachelor an Fachhochschulen 
- Masterabschluss

Master an Universitäten (Abschlussprüfung vorausgesetzt)

Master an Fachhochschulen (Abschlussprüfung vorausgesetzt) - Sonstiger Abschluss

Abschlusszeugnis/Zertifikat

Die Autoren betrachten es als Versäumnis der mathematischen und fachdidaktischen Fachgesellschaften, sich bei Beginn der Bachelor-Master-Reform nicht länderübergreifend auf weniger Abschlüsse verständigt und diese mit den Wissenschafts- respektive Kultusministerien der Länder abgestimmt zu haben. Wir meinen, hier dringenden Handlungsbedarf konstatieren zu müssen. Überdies sind an einzelnen Standorten Studiengänge entstanden, die formal keine Lehramtsstudiengänge sind, aber nicht automatisch dem Anspruch genügen, ein mathematisches Fachstudium, spätestens in der Master-Phase zu ermöglichen.

\section{I.2 Das Problem der Mehrfach-Bachelor-Studiengänge ohne Lehramtsoption}

Bachelor-Studien sollen nach politischer Vorgabe in der Regel polyvalent angelegt werden. Diesem Axiom musste gefolgt werden. Es existieren an einzelnen Universitäten Zweifach- oder sogar Mehrfach-Bachelor mit dem Basisfach Mathematik und - von Hochschule zu Hochschule unterschiedlich - einem Zweitfach, das generell nicht unbedingt mathematikaffin ist. Das mag zunächst plausibel erscheinen, wenn man an einen Lehramtsabschluss denkt. Einzelne Studiengänge sind mit dem Prädikat ,ohne Lehramtsoption" versehen, sehen also keine parallelen erziehungswissenschaftlichen Begleitstudien vor - unter der illusionären Annahme, dass der Arbeitsmarkt für solchermaßen Qualifizierte Platz bietet. Dieser Auffassung neigen auch die Rektorate meistens zu. Möglicherweise wollte man auch dem Fall vorbeugen, dass die Aufnahme am Numerus Clausus im ErziehungswissenschaftenBegleitstudium scheitert.

Kritisch sehen wir allerdings den Umstand, dass Mathematikorientierung sehr unterschiedlich verstanden wird. Im Fall der an der Universität Münster angebotenen Studiengänge beinhalten Studienpläne allerdings Themen, die dem Haupt- und Realschulbereich zuzuordnen sind.

Universitäten, an denen keine Lehrämter ausgebildet werden, verfolgen andere, zum Teil sehr gelungene Konzepte wie z. B. den Doppelstudiengang BSc Mathematik/ Informatik an der Heinrich-Heine-Universität in Düsseldorf, der einen um ein Semester zeitversetzten Parallelstudiengang Informatik beinhaltet und eine Regelstudienzeit von 7 Semestern aufweist.

Die hohen Frauenanteile ( $>60 \%$ ) deuten darauf hin, dass die Mehrfach-Bachelor-Studiengänge (mit Lehramtsoption) als sehr „lehramtsnah“ einzustufen sind, wobei die Lehramtsoption daraus noch keinen Lehramtsstudiengang macht.

Es scheint den Autoren an der Zeit, dass eine bundesweite Diskussion über Mehrfach-Bachelor-Studiengänge ohne
Tabelle I. Studienanfänger im Studienjahr 2014 im Studienfach Mathematik nach Hochschulen, nur „Mehr-Fächer-Bachelor ohne Lehramtsoption"

\begin{tabular}{|c|c|c|c|c|c|c|}
\hline \multirow{3}{*}{$\begin{array}{l}\text { Hochschule } \\
(\mathrm{U}, \mathrm{TU}, \mathrm{TH})\end{array}$} & \multicolumn{6}{|c|}{ Studienanfänger im ... } \\
\hline & \multicolumn{3}{|c|}{$\begin{array}{l}\text { I. Hochschul- } \\
\text { semester }\end{array}$} & \multicolumn{3}{|c|}{$\begin{array}{l}\text { I. Fach- } \\
\text { semester }\end{array}$} \\
\hline & $\mathrm{i}$ & $\mathrm{m}$ & w & $\mathrm{i}$ & $\mathrm{m}$ & w \\
\hline LMU München & 81 & 51 & 30 & 117 & 72 & 45 \\
\hline HU Berlin & 8 & 4 & 4 & 9 & 4 & 5 \\
\hline Göttingen & 4 & 2 & 2 & 5 & 2 & 3 \\
\hline Bielefeld & 176 & 90 & 86 & 323 & 160 & 163 \\
\hline Bochum & 15 & 10 & 5 & 15 & 10 & 5 \\
\hline Münster & - & 一 & - & I & - & I \\
\hline Wuppertal & 31 & 23 & 8 & 49 & 33 & 16 \\
\hline Koblenz-L. & - & 一 & - & 4 & 4 & - \\
\hline Gesamt & 315 & 180 & 135 & 523 & 285 & 238 \\
\hline
\end{tabular}

Lehramtsoption angestoßen wird und intelligente Konzepte zur Nachahmung erarbeitet werden.

Das statistische Bundesamt hat $u$. a. die in Tabelle I aufgeführten Zahlen zu Mehrfach-Bachelor-Studiengängen ermittelt. Die Tabelle zeigt, dass Destatis die Studierenden doppelt erfasst: einmal nach der Anzahl der Fachsemesters bezogen auf den Studiengang, in dem sie sich befinden, und gleichzeitig auch mit Blick auf das Hochschulsemester, das sie gerade besuchen. Man kann erkennen, wie viele Semester die Studierenden an deutschen Hochschulen insgesamt - unabhängig von den gewählten Studiengängen - bisher eingeschrieben gewesen sind. Nach der Umsetzung der Bologna-Reform gibt es daher zwei Typen von Anfängern: Studierende, die sich im ersten Fachsemester und gegebenenfalls auch im ersten Hochschulsemester eines Bachelorstudiengangs befinden sowie Studierende im ersten Fachsemester eines Masterstudiengangs, aber - bis auf universitäre Neuzugänge - dann nicht mehr im ersten Hochschulsemester.

Aufgrund des aktuellen Mathematiklehrermangels werden derzeit verstärkt Seiteneinsteiger angeworben, welche "Nichtqualifizierungen“" auch immer ihr Abschlusszeugnis aufzuweisen hat. Irgendwie glaubt die Administration diese Defizite kompensieren zu können. Im Hinblick auf nach unserer Meinung nach qualifizierenden Mehrfach-Bachelor (ohne Lehramtsoption) hielten wir es intellektuell für geboten, potentielle Interessenten offen über die Einschränkungen zu informieren.

\section{Studienanfänger im STB Mathematik}

\section{I Studienanfängerzahlen (Bachelor/ Master) 2014}

In Tabelle 2 stellen wir die Studienanfängerzahlen des Studienbereichs Mathematik im Studienjahr 2014 dar. $^{4}$

Rund 40000 Studierende streben aktuell einen universitären Abschluss in Mathematik außerhalb des Lehramtsbereichs (!) an. Rund 7000 Schulabgänger konnten für einen Hochschulstart im Bereich Mathematik gewonnen werden. Knapp 13000 Studierende befanden sich 
Tabelle 2. STB Mathematik: Studienanfängerzahlen im Studienjahr 2014 (Quelle: [4], Seite 244)

\begin{tabular}{|c|c|c|c|c|}
\hline & \multicolumn{2}{|c|}{ Univ. Abschluss } & \multicolumn{2}{|c|}{ darunter } \\
\hline & & & Bachelor & Master \\
\hline \multicolumn{5}{|c|}{ Fachsemesterzählung } \\
\hline & insg. & I.u.2. FS & I.u.2. FS & I.u.2. FS \\
\hline $\mathrm{m}$ & 24.543 & 8.897 & 6.959 & 1.847 \\
\hline w & 15.664 & 6.735 & 5.876 & 798 \\
\hline $\mathrm{i}$ & 40.207 & I 5.632 & 12.835 & 2.645 \\
\hline \multicolumn{5}{|c|}{ Hochschulsemesterzählung } \\
\hline & insg. & I.u.2. HS & I.u.2. HS & I.u.2. HS \\
\hline $\mathrm{m}$ & 24.543 & 4.147 & 3.930 & 155 \\
\hline w & 15.664 & 3.081 & 2.958 & 92 \\
\hline $\mathrm{i}$ & 40.207 & 7.228 & 6.888 & 247 \\
\hline
\end{tabular}

2014 in den ersten beiden Fachsemestern eines BachelorStudiengangs, rund 2600 in den ersten beiden Fachsemestern eines Master-Studienganges. Gleichwohl verwundern Zahlendifferenzen in der Tabelle: nämlich die unterschiedlichen Ergebnisse bei den Fachsemester- respektive Hochschulsemester-Zählungen. Dass von den 2645 Studienanfängern Master (I./2. Fachsemester) sich 247 Personen im I./2. Hochschulsemester befinden, überrascht nicht. Hier sollte es sich um Studierende handeln, die erstmals in Deutschland eingeschrieben sind. 6888 Studierende im I./2. Hochschulsemester haben sich in ein Bachelor-Studium eingeschrieben; aber die reale Zahl der Hörer in den ersten beiden Semestern beträgt fast 13000 . Fast die Hälfte dieser Studierenden muss zuvor an anderer Stelle in Hochschulen eingeschrieben gewesen sein.

Was wissen wir über diese nicht unbeträchtliche Klientel von „Späteinsteigern“ unserer Anfängervorlesungen, also jenen vielen Studierenden, die in einem höheren Hochschulsemester ins Mathematikstudium einsteigen? Unterscheidet sich diese Klientel von den „Frischeinsteigern“?

Es könnte sein, dass wegen eines Numerus Clausus (NC) Studierende „parken“ müssen. Leider liegen uns keine erschöpfenden Informationen über NC-Beschränkungen an deutschen Universitäten für das Fach Mathematik vor. Die Universität Bonn hat uns dazu interessante Daten zur Kenntnis gebracht. Die Beschränkung beim Bachelor Mathematik lag dort im Jahr 2015 bei 228 Studierenden, für das Lehramt lautete der Parameter 100. Im Studienjahr 2015 haben sich insgesamt 644 bzw. 618 Interessenten beworben; auf der Basis früherer Erfahrungen hat man alle Interessenten zugelassen und selbst nach mehreren Auswahlprozessen blieben noch wenige Plätze unbesetzt. Kann es hier der Fall sein, dass in den Werten für Studienanfänger nach Fachsemesterbetrachtung auch ,parkende" Studierende (ohne mathematisches Interesse) enthalten sind? Dies bleibt jedoch Spekulation.

Die Tabelle 2 macht einen weiteren Aspekt deutlich, der sich allerdings nicht ganz einfach erklären lässt: Unterstellt man, dass die Anfängerzahlen über die Studienjahre annähernd konstant bleiben, so erkennt man, dass die Zahl der Master-Anfänger fast nur ein Viertel der Bachelor-Anfänger beträgt. Verlieren wir wirklich rund $75 \%$ unserer Anfänger? Diese Kennwerte bedürften einer weiteren Analyse.

\subsection{Studienanfängerzahlen 2014 nach Hochschulen}

Das Statistische Bundesamt hat uns eine standortspezifische Auflistung der Studienanfängerzahlen für das Jahr 2014 zur Verfügung gestellt (vgl. Tabelle 3 auf S. 238). Bei der Abfrage wurde explizit das Studienziel Lehramt ausgeschlossen.

Alle Zahlen basieren auf den Einschreibedaten der Studierenden und berücksichtigen keine Umorientierung nach Semesterbeginn. Die Zahlen sind offiziell erhobene Kenngrößen, die über die statistischen Landesämter zu Destatis weitergeleitet wurden. Die Zahlen der Anfänger im I./2. Hochschulsemester - weil für den Studienbereich Mathematik erfasst - sind auch in den Zahlen der Erstsemester gemäß der Fachsemesterzählung enthalten.

Was fällt auf?

I. Rückfragen bei einzelnen Kollegen/ Kolleginnen haben ergeben, dass diese Zahlen teilweise als überhöht angesehen werden müssen. Man sollte allerdings vorsichtig sein, die Destatis-Zahlen in Frage zu stellen. Defizite der Datenerhebung liegen zumeist auf der lokalen Ebene.

2. Die Zahlen für Düsseldorf und die TU Berlin fallen besonders hoch aus. Vor Ort vermutet man, dass möglicherweise andere Studierende im NC-freien Studiengang Mathematik ,parken“.

3. Die Liste enthält Standorte, die bekanntlich keine eigenständige Mathematikerausbildung betreiben, u.a. Flensburg, Hildesheim, Vechta. Auffällig ist der hohe Frauenanteil an diesen Standorten. Eventuell greift hier unsere Erklärung, dass Mehrfach-Bachelor ohne Lehramtsoption gezählt wurden.

Formal ist dies richtig, doch die Mehrfach-BachelorStudiengänge ohne Lehramtsoption scheinen unsere Zahlen zu „schönen“. Auch wenn Düsseldorf mit seinem gekoppelten Zweifach-Bachelor-Studiengang Mathematik/Informatik ein interessantes Konzept verfolgt, die hohe Zahl von mehr als I 500 Erstsemestern lässt sich dadurch nicht erklären.

4. Der hohe Frauenanteil bei einzelnen Hochschulen überrascht (z. B. Braunschweig, Oldenburg, Passau, Potsdam, Siegen, Trier, Wuppertal). Das kennt man eigentlich nur von den ,kleinen“ Lehrämtern. Insofern wurden hier wieder (vgl. Punkt 3) auch Studierende mit einem Mehrfach-Bachelor erfasst, wie uns Destatis für die Daten von Braunschweig belegen konnte. In einem anderen Projekt, in dem wir BachelorAbsolventen (2015) ermitteln, haben wir mittlerweile erkannt, dass wir unbedingt die fachfernen MehrfachBachelor (mit bzw. ohne Lehramtsoption) herausrechnen müssen; allerdings sind solche Recherchen bei Destatis kostenpflichtig. 
Einige Fragen konnten wir beantworten, andere blieben unbeantwortet; hierzu müsste man noch detaillierter in das Datenmaterial schauen. Entsprechende Anfragen an Destatis sind allerdings kostenpflichtig. Es wäre einiges gewonnen, wenn jeder Dekan oder geschäftsführende Direktor die weitergemeldeten Zahlen bewusst zur Kenntnis nehmen und sie vielleicht direkt und unverzüglich der Konferenz mathematischer Fachbereiche (KMathF) melden würde. Die uns vorliegende letzte Liste der KMathF enthält für manchen Hochschulstandort einen leeren Eintrag.

Sollten diese Zahlen nicht stimmen, so sind auch alle Ableitungen und Erklärungen zweifelhaft; dann können wir den Vorwurf, dass wir hohe Abbruchquoten verursachen, nicht widerlegen. Es liegt also im Interesse unseres Faches, dass die vorliegenden Zahlen korrekt und offen zugänglich sind. Es erscheint den Autoren dringend ratsam, durch konzertierte Aktionen die Zahl der unterschiedlichsten Angebote im Lehramtsbereich auszudünnen. Wir sind uns allerdings der Eigeninteressen an manchen Standorten bewusst. Gerne würden wir auch eine ehrliche Diskussion über die Zweifach-BachelorStudiengänge ohne Lehramtsoption anregen, um von erfolgreichen Ansätzen lernen zu können und umgekehrt eine gewisse Ähnlichkeit der Konzepte zu initiieren. Von den Akkreditierungsagenturen scheint allerdings bislang kein Impuls auszugehen. Schließlich wäre es sehr hilfreich, wenn man mehr Einsichten über die Erfahrungen mit NC-beschränkten Studiengängen erhalten könnte. Die im Text zitierten Zahlen aus Bonn haben uns überrascht.

Wir danken Stefan Brings und Thomas Feuerstein vom Statistischen Bundesamt für hilfreiche und ergänzende Hinweise bei der Erstellung dieses Textes.

\section{Anmerkungen}

I. https://www.uni-due.de/mathematik/agtoerner/zahlen.shtml 2. http://www.studis-online.de

3. Prüfungen werden bei Destatis in sogenannten Prüfungsjahren erfasst; hierbei werden jeweils die Zahlen eines Wintersemesters und des darauf folgenden Sommersemesters zusammengefasst.

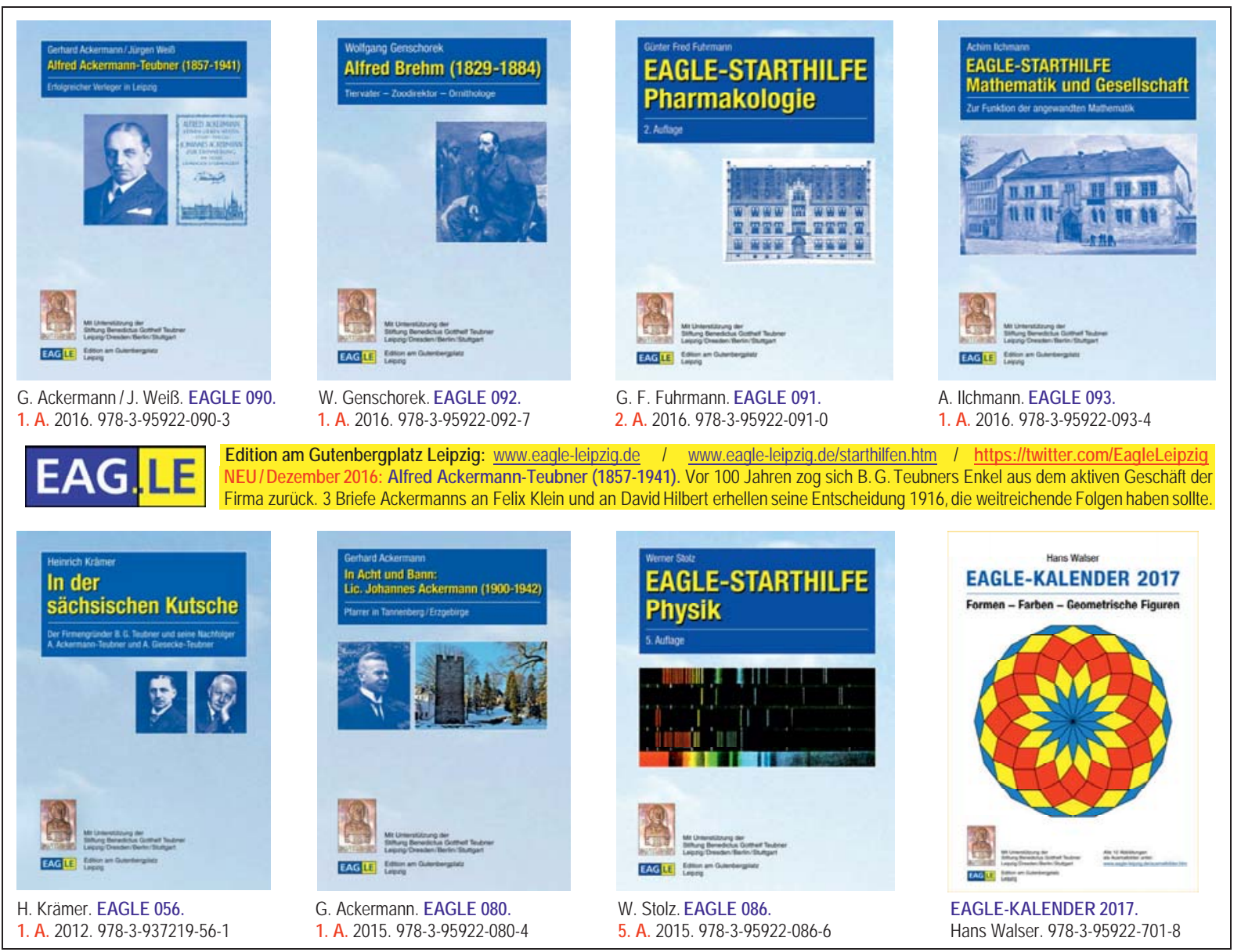


Tabelle 3. Studienanfänger im Studienjahr 2014 im Studienfach Mathematik nach Hochschulen

\begin{tabular}{|c|c|c|c|c|c|c|}
\hline \multirow{3}{*}{$\begin{array}{l}\text { Hochschule } \\
(\mathrm{U}, \mathrm{TU}, \mathrm{TH})\end{array}$} & \multicolumn{6}{|c|}{ Studienanfänger im ... } \\
\hline & \multicolumn{3}{|c|}{$\begin{array}{l}\text { I. Hochschul- } \\
\text { semester }\end{array}$} & \multicolumn{3}{|c|}{$\begin{array}{l}\text { I. Fach- } \\
\text { semester }\end{array}$} \\
\hline & $\mathrm{i}$ & $\mathrm{m}$ & $w$ & $\mathrm{i}$ & $\mathrm{m}$ & w \\
\hline Aachen & 128 & 82 & 46 & 250 & 167 & 83 \\
\hline Augsburg & 74 & 43 & 31 & 149 & 86 & 63 \\
\hline Bayreuth & 43 & 28 & 15 & 84 & 54 & 30 \\
\hline FÚ Berlin & $13 \mid$ & 75 & 56 & 338 & 176 & 162 \\
\hline TU Berlin & 247 & 139 & 108 & 900 & 443 & 457 \\
\hline HU Berlin & 170 & 107 & 63 & 317 & 197 & 120 \\
\hline Bielefeld & 181 & 92 & 89 & 357 & 185 & 172 \\
\hline Bochum & 152 & 82 & 70 & 220 & $13 \mid$ & 89 \\
\hline Bonn & 207 & 160 & 47 & 320 & 255 & 65 \\
\hline Braunschweig & 100 & 30 & 70 & 150 & 58 & 92 \\
\hline Bremen & 48 & 30 & 18 & 116 & 71 & 45 \\
\hline Jacobs U Bremen & 10 & 9 & I & 14 & 11 & 3 \\
\hline Chemnitz & 27 & 19 & 8 & 49 & 36 & 13 \\
\hline Clausthal & 3 & 2 & I & 4 & 2 & 2 \\
\hline Cottbus & II & 4 & 7 & 32 & 9 & 23 \\
\hline Darmstadt & 136 & 99 & 37 & 247 & 177 & 70 \\
\hline Dresden & 84 & 53 & 31 & 125 & 88 & 37 \\
\hline Dortmund & 86 & 45 & 41 & 180 & 88 & 92 \\
\hline Düsseldorf & 347 & 135 & 212 & 1.549 & 576 & 973 \\
\hline DuE & 124 & 65 & 59 & 418 & 199 & 219 \\
\hline Eichstädt & II & 4 & 7 & 17 & 7 & 10 \\
\hline Erlangen-Nürnberg & 71 & 43 & 28 & 139 & 93 & 46 \\
\hline Flensburg & 91 & 24 & 67 & 105 & 31 & 74 \\
\hline Frankfurt & 182 & 107 & 75 & 573 & 315 & 258 \\
\hline Freiburg & 77 & 54 & 23 & 163 & 117 & 46 \\
\hline Gießen & 35 & 19 & 16 & 180 & 80 & 100 \\
\hline Göttingen & 118 & 78 & 40 & 220 & 155 & 65 \\
\hline Greifswald & 34 & 17 & 17 & 91 & 48 & 43 \\
\hline Hagen & 119 & 82 & 37 & 598 & 429 & 169 \\
\hline Hannover & 183 & 103 & 80 & 303 & 172 & $13 \mid$ \\
\hline Halle & 21 & 14 & 7 & 29 & 19 & 10 \\
\hline Hamburg & 103 & 63 & 40 & 186 & 112 & 74 \\
\hline Heidelberg & 150 & 98 & 52 & 301 & 206 & 95 \\
\hline
\end{tabular}

\begin{tabular}{|c|c|c|c|c|c|c|}
\hline \multirow{3}{*}{$\begin{array}{l}\text { Hochschule } \\
(\mathrm{U}, \mathrm{TU}, \mathrm{TH})\end{array}$} & \multicolumn{6}{|c|}{ Studienanfänger im .. } \\
\hline & \multicolumn{3}{|c|}{$\begin{array}{l}\text { I. Hochschul- } \\
\text { semester }\end{array}$} & \multicolumn{3}{|c|}{$\begin{array}{l}\text { I. Fach- } \\
\text { semester }\end{array}$} \\
\hline & i & $\mathrm{m}$ & $w$ & $\mathrm{i}$ & $\mathrm{m}$ & w \\
\hline Hildesheim & 123 & 40 & 83 & 152 & 59 & 93 \\
\hline Imen. & 12 & 7 & 5 & 24 & 14 & 10 \\
\hline Jena & 21 & 16 & 5 & 44 & 33 & 11 \\
\hline Kaiserslautern & 129 & 88 & $4 I$ & 199 & 138 & 61 \\
\hline KIT & 122 & 80 & 42 & 182 & 122 & 60 \\
\hline Kassel & 39 & 21 & 18 & 81 & 45 & 36 \\
\hline Kiel & 59 & 36 & 23 & 117 & 69 & 48 \\
\hline Köln & 104 & 66 & 38 & 186 & 117 & 69 \\
\hline Konstanz & 48 & 27 & 21 & 66 & $4 I$ & 25 \\
\hline Leipzig & 47 & 38 & 9 & 87 & 55 & 32 \\
\hline Lübeck & 52 & 18 & 34 & 89 & 31 & 58 \\
\hline Magdeb. & 30 & 18 & 12 & 60 & 38 & 22 \\
\hline Mainz & 74 & 45 & 29 & $|3|$ & 78 & 53 \\
\hline Marburg & 38 & 26 & 12 & 264 & 137 & 127 \\
\hline U München & 90 & 58 & 32 & 193 & 124 & 69 \\
\hline TU München & 180 & 124 & 56 & 314 & 221 & 93 \\
\hline Münster & 182 & 107 & 75 & 282 & 178 & 104 \\
\hline Oldenburg & 156 & 63 & 93 & 221 & 99 & 122 \\
\hline Osnabrück & 54 & 32 & 22 & 81 & 49 & 32 \\
\hline Paderborn & 42 & 24 & 18 & 74 & 43 & 31 \\
\hline Passau & 29 & 16 & 13 & 56 & 30 & 26 \\
\hline Potsdam & 27 & 14 & 13 & 105 & 46 & 59 \\
\hline Regensburg & 73 & 48 & 25 & 163 & 113 & 50 \\
\hline Rostock & 23 & 16 & 7 & 60 & 36 & 24 \\
\hline Saarbrücken & 31 & 24 & 7 & 89 & 61 & 28 \\
\hline Siegen & 46 & 17 & 29 & 160 & 64 & 96 \\
\hline Stuttgart & 66 & 37 & 29 & 102 & 66 & 36 \\
\hline Trier & 25 & 9 & 16 & 51 & 28 & 23 \\
\hline Tübingen & 51 & 34 & 17 & 94 & 67 & 27 \\
\hline Ulm & 90 & 47 & 43 & 186 & 99 & 87 \\
\hline Vechta & 93 & 25 & 68 & 117 & 35 & 82 \\
\hline Würzburg & 81 & 51 & 30 & 189 & 116 & 73 \\
\hline Wuppertal & $12 \mid$ & 66 & 55 & 330 & 172 & 158 \\
\hline
\end{tabular}

4. Studierende werden bei Destatis in sogenannten Studienjahren gezählt; dabei werden jeweils ein Sommersemester und das darauf folgende Wintersemester zusammengefasst.

\section{Literatur}

[I] Destatis 2012. Hochschulen auf einen Blick, Ausgabe 2012. Wiesbaden: Statistisches Bundesamt.

[2] Destatis 2013. Hochschulen auf einen Blick, Ausgabe 2013. Wiesbaden: Statistisches Bundesamt.

[3] Destatis 2014. Bildung und Kultur; Prüfungen an Hochschulen. Fachserie II, Reihe 4.2. Wiesbaden: Statistisches Bundesamt.

[4] Destatis 2014. Bildung und Kultur; Studierende an Hochschulen. Fachserie II, Reihe 4.I. Wiesbaden: Statistisches Bundesamt.

[5] Destatis 2016. Bildung und Kultur. Schnellmeldungsergebnisse der Hochschulstatistik zu Studierenden und Studienanfänger/innen - vorläufige Ergebnisse - Wintersemester 2015/16. Wiesbaden: Statistisches Bundesamt.

[6] Destatis 2016. Hochschulen auf einen Blick - 2016. Wiesbaden: Statistisches Bundesamt.

[7] Dieter, M.; Brugger, P.; Schnelle, D. \& Törner, G. (2008). Zahlen rund um das Mathematikstudium - Teil I. Mitteilungen der Deutschen Mathematiker-Vereinigung (DMV) 16 (I), 42-47.

[8] Dieter, M.; Brugger, P.; Schnelle, D. \& Törner, G. (2008). Zahlen rund um das Mathematikstudium - Teil 2. Mitteilungen der Deutschen Mathematiker-Vereinigung (DMV) I6 (2), I06-I I0.

Britta Berndtsen, Dr. Miriam Dieter und Prof. Dr. Günter Törner, Fakultät für Mathematik, Universität Duisburg-Essen, 45I 17 Essen britta.berndtsen@uni-due.de miriam.dieter@uni-due.de guenter.toerner@uni-due.de

Günter Törner arbeitet fachwissenschaftlich seit vielen Jahren in der nichtkommutativen Ringtheorie; zugleich ordnet er sich mit zahlreichen Beiträgen in den Bereich mathematics education ein. In den letzten fünf Jahren war er als Vorstandsmitglied maßgeblich am Aufbau des in Berlin ansässigen Deutschen Zentrums für Lehrerbildung Mathematik (DZLM) beteiligt.

Miriam Dieter hat über das Thema „Abbruchquoten während des Mathematikstudiums" geforscht und arbeitet derzeit als Dekansassistentin an der Universität Duisburg-Essen.

Britta Berndtsen ist nichtwissenschaftliche Mitarbeiterin an der Fakultät für Mathematik der Universität Duisburg-Essen.
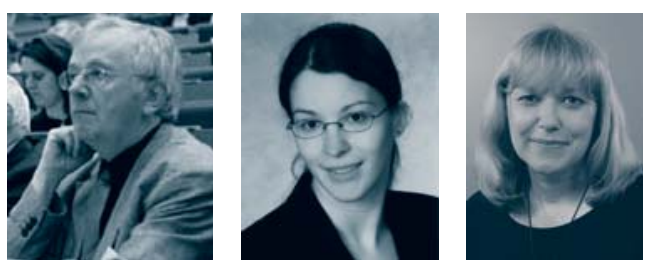

Günter Törner, Miriam Dieter, Britta Berndtsen (v. I. n. r.) 